VOL. $20(1979), 57-70$.

\title{
Duality for finite abelian \\ hypergroups over splitting fields
}

\section{J.R. McMullen and J.F. Price}

\begin{abstract}
A duality theory for finite abelian hypergroups over fairly general fields is presented, which extends the classical duality for finite abelian groups. In this precise sense the set of conjugacy classes and the set of characters of a finite group are dual as hypergroups.
\end{abstract}

\section{Introduction}

This paper is a companion to McMullen [5] which should be consulted for the background to the concept of a hypergroup as well as for details of notation and definitions. These are used below without explanation.

Throughout $H$ will denote a finite abelian hypergroup with coefficients in a field $k$ with multiplicity, degree, and conjugation functions denoted by $n: H^{3} \rightarrow k, d: H \rightarrow k \backslash\{0\}$, and $-: H \rightarrow H$ respectively. Its identity will be denoted by 1 .

The aim of this paper is to prove, for fairly general fields $k$, a duality theory which extends the classical duality for finite abelian groups. As a consequence, the set of characters and the set of conjugacy classes of a finite group are dual as hypergroups. The procedure will be to first view a hypergroup as a distinguished subset of a hypergroup algebra or double algebra as explained in [5] and then to examine duals of such algebras.

Received 8 November 1978. 


\section{Duals of double algebras}

Let $k H$ be the free $k$-module with basis $H$. Then, as explained in Proposition 1 of [5], $k H$ can be made into a double algebra in a natural manner so that $\left\{d(a)^{-1} a \mid a \in H\right\}$ is the set of grouplikes in $k H$. Since $k H$ is a cogebra, its dual $(k H)^{*}=\operatorname{hom}_{k}(k H, k)$ becomes an algebra when a multiplication $M^{*}:(k H)^{*} \otimes(k H)^{*} \rightarrow(k H)^{*}$ and a unit $u^{*}: k \rightarrow(k H)^{*}$ are defined as the $k$-linear extensions of

$$
\begin{aligned}
M^{*}(f \otimes g)(a) & =d(a)^{-1} f(a) g(a), \\
u^{*}(\alpha)(a) & =\alpha d(a),
\end{aligned}
$$

where $f, g \in(k H)^{*}, a \in H$, and $\alpha \in k$. (Recalling that $\Delta: k H \rightarrow k H \otimes k H$ is the comultiplication on $k H$ defined by

$$
\Delta(a)=d(a)^{-1} a \otimes a \text { for } a \in H,
$$

$M^{*}(f \otimes g)$ is the map which makes

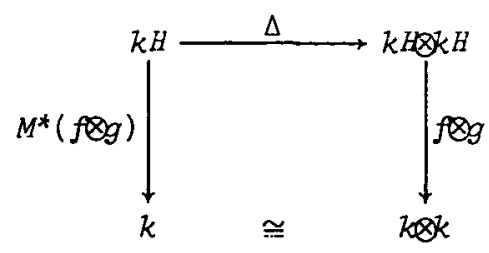

commute.)

Now define, in a similar fashion, a comultiplication

$\Delta^{*}:(k H)^{*} \rightarrow(k H)^{*} \otimes(k H)^{*}$ and a counit $d^{*}:(k H)^{*} \rightarrow k$ on $(k H)^{*}$ by

$$
\begin{aligned}
\Delta^{*}(f)(a \otimes b) & =f(a \cdot b), \\
d^{*}(f) & =f(1),
\end{aligned}
$$

for $f \in(k B)^{*}$ and $a, b \in H$. (Actually (4) defines $\Delta^{*} f$ as an element of $\left(k H \otimes k^{H}\right)^{*}$, but since $H$ is finite, this can be identified with $(k H)^{*} \otimes(k H)^{*}$.) Finally define a map $S^{*}:(k H)^{*} \rightarrow(k H)^{*}$ as the $k$-linear extension of

$$
\left(S^{*} f\right)(a)=f(S a)=f(\bar{a}),
$$

where $f \in(k H)^{*}$ and $a \in H$. In practice we usually write $S^{*} f$ as $\bar{f}$. Our intention is to give conditions on $k$ under which $\left(k^{H}\right)^{*}$ with the above maps is a double algebra [5]. Already we have from some routine 
manipulations :

\section{Proposition}

The linear space $(k H)^{*}$ is a $k$-algebra with wonit $u^{*}$, a $k$-cogebra with counit $d^{*}, u^{*}$ is a cogebra map, $d^{*}$ is an algebra map, and $S^{*}$ is both an algebra and a cogebra map.

It remains to

(i) give conditions on $k$ which ensure that the grouplikes in $(k H)^{*}$ are spanning, and

(ii) verify $[5$, Axiom $(\mathrm{A} 7)]$ for $(k H)^{*}$.

\section{Theorem}

Consider the following conditions on an abelian hypergroup $H$ of finite cardinality $s$ with coefficients in $k$ :

(i) $k H$ is the direct sum of $s$ ideals;

(ii) $k H$ has $s$ distinct algebra homomorphisms into $k$;

(iii) the grouplikes in $(k H)^{*}$ are a spanning set;

(iv) each $T_{a}: \xi \mapsto a \xi \quad(a \in H)$ as an element of $\operatorname{end}_{k}(k H)$ has its eigenvalues in $k$.

Then conditions (i), (ii), and (iii) are equivalent and imply (iv). Condition (iv) implies the first three conditions when $k$ is a subfield of (c.

Recalling that grouplikes are linearly independent [7, Proposition 3.2.1 (b)] the equivalence of (ii) and (iii) follows from the following lemma (which has as a consequence that $d: k H \rightarrow k$ is grouplike).

\section{Lemma}

Any member of $(k H)^{*}$ is grouplike if and only if it is an algebra map.

Proof. Given nonzero $f$ in $(k H)^{*}$, then by definition it is grouplike if and only if $\Delta^{*} f=f \otimes f$, which is the case if and only if $\left(\Delta^{*} f\right)(a \otimes b)=(f \otimes f)(a \otimes b)$ for all $a, b \in H$. But by (4) this identity occurs precisely when $f(a . b)=f(a) f(b)$; that is, when $f$ is 
an algebra map.

Proof of Theorem 4. The equivalence of ( $i i)$ and ( $i i i)$ is settled by the preceding lemma while the implication $"(i) \Rightarrow(i i) "$ is trivial. Now assume $(i i)$ and $(i i i)$ so that we have $s$ algebra homomorphisms $\rho_{1}, \ldots, \rho_{s}$ from $k H$ into $k$ which form a basis for $(k H)^{*}$. Hence the $\operatorname{map} \rho: k H \rightarrow k \oplus \ldots \oplus k$ (s times) given by $\rho(\xi)=\left(\rho_{1}(\xi), \ldots, \rho_{s}(\xi)\right)$ is an algebra monomorphism and so an isomorphism. Thus $(i)$ is satisfied.

We will now show that (ii) implies (iv). Assume (ii) and let $\rho_{1}, \ldots, \rho_{s}$ denote the algebra homomorphisms in $(k H)^{*}$. For each $i \in\{1, \ldots, s\}$ put

$$
x_{i}=\sum_{j=1}^{\varepsilon} \rho_{i}\left(a_{j}\right) r\left(a_{j}\right)^{-1} a_{j},
$$

where $H=\left\{a_{1}, \ldots, a_{s}\right\}$. Notice that $x_{i}$ remains the same if we transform the basis $\left\{a_{1}, \ldots, a_{s}\right\}$ by a diagonal matrix. Since the $\bar{a}_{j}$ and the $\rho_{i}$ are bases for $k H$ and $(k H)^{*}$ respectively, the set $\left\{x_{1}, \ldots, x_{s}\right\}$ is $k$-linearly independent and in particular contains no zero elements.

An easy calculation shows that

$$
T_{a} x_{i}=a x_{i}=\rho_{i}(a) x_{i} \quad(a \in H),
$$

whence $\rho_{i}(\alpha), i=1, \ldots, s$, are eigenvalues of $T_{a}$ with corresponding eigenvectors $x_{1}, \ldots, x_{s}$. These eigenvectors are a basis for $k H$ indicating that we have found all the eigenvalues of $T_{a}$. Thus (iv) is satisfied.

To prove that (iv) implies (ii) under the extra assumption that $k$ is a subfield of $\mathbb{a}$, let $\left\{\rho_{1}, \ldots, \rho_{s}\right\}$ be the distinct complex algebra homomorphisms of $\mathbb{C} H$. (These exist since $\mathbb{\downarrow}$ is algebraically closed and a $H$ is semisimple [6].) From (8) we know that the eigenvalues of $T_{a} \in \operatorname{end}_{\mathbb{C}}(\mathbb{C} H)$ are $\rho_{i}(a), i=1, \ldots, s$. But $T_{a}$ can also be considered as an element of $\operatorname{end}_{k}(k H)$, still with eigenvalues 
$\rho_{1}(a), \ldots, \rho_{s}(a)$, and so by hypothesis the $\rho_{i}$ take their values in $k$.

\section{Splitting fields}

A field $k$ is called a splitting field for a finite abelian hypergroup $H$ if $k H$ satisfies any of the conditions (i)-(iii) of Theorem 4.

REMARKS. 1. If $H$ has coefficients in $k \subseteq \mathbb{a}$, then the smallest splitting field for $H$ is clearly obtained by adjoining the elements $\left\{\rho_{i}\left(a_{j}\right) \mid 1 \leq i, j \leq s\right\}$ to $k$.

2. Let $H$ have integral coefficients. Then the matrices of the transformations $T_{a}(a \in H)$ have integral entries, and hence the complex numbers $\rho(a) \quad(a \in H, \rho: \mathbb{Q} \rightarrow \mathbb{G}$ a complex homomorphism) are algebraic integers.

\section{Structure constants}

Let $H=\left\{a_{1}, \ldots, a_{s}\right\}$ be a commutative hypergroup with coefficients in a splitting field $k$ and denote the grouplikes in $(k H)^{*}$ by $\left\{d=\rho_{1}, \rho_{2}, \ldots, \rho_{s}\right\}$. The stmucture constants of $H$ (or of $k H$ ) are defined as

$$
c_{i}=\sum_{m=1}^{s} r\left(a_{m}\right)^{-1} \rho_{i}\left(a_{m}\right) \rho_{i}\left(\bar{a}_{m}\right) \quad(i=1, \ldots, s) .
$$

Notice that $c_{1}=\|H\|$ by definition (see 1.2 of [5]).

$$
\text { Since } r(\alpha a)=\alpha^{2} r(a) \text { for } \alpha \in k \text { and } a \in H \quad[5 \text {, proof of }
$$

Proposition 2], it is seen that, up to permutations, the structure constants depend only upon $k H$ as a double algebra. In particular,

$$
c_{i}=\sum_{a \in H_{1}} \rho_{i}(a) \rho_{i}(\bar{a}) \quad(i=1, \ldots, s),
$$

where $H_{1}$ denotes the set of standard elements in $k H$ (assuming that $r\left(a_{i}\right)$ is a square in $k$ for each $\left.i\right)$. In the case when $H$ is the character hypergroup of a finite group $G$, the accompanying field $k$ being a splitting field for $G$ with the characteristic of $k$ not dividing 
the order of $G$, the structure constants have the property that the numbers $h_{i}=c_{1} c_{i}^{-1}(i=1, \ldots, s)$ are the cardinalities of the conjugacy classes of $G[2,(31.13)]$.

We now turn to the problem of giving conditions which ensure that the structure constants are nonzero, an hypothesis used in the proof of the duality Theorem 12. Assume that $k$ is a subfield of $\mathbb{C}$ and define an inner product on $k H$ by putting

$$
\langle a, b\rangle=|r(a)| \delta_{a b}
$$

and extending by sesquilinearity.

PROPOSITION. The following conditions on a finite hypergroup $H$ with coefficients in a complex splitting field $k$ are equivalent:

(i) under the above inner product, the adjoint of

$$
T_{a}: b \mapsto a \cdot b \text { is } T_{\bar{a}} \text {; }
$$

(ii) $n(a, b, c)$ is real for $a l z a, b, c$ in $H$;

(iii) $\rho(\bar{a})=\overline{\rho(a)}$ for $a \in H$ and grouplikes $\rho$.

Moreover, these conditions imply that the structure constants are strictly positive real numbers.

Proof. The proof of the equivalence of conditions (i), (ii), and (iii) is routine and follows from the identity $n(a, b, c)|r(c)|=(a \cdot b \quad c)$. To complete the proof, suppose that (iii) is satisfied and choose a square root $r(a)^{\frac{3}{2}} \in \mathbb{Q}$ for each $a \in H$. Then working in $\mathbb{C} H$ we obtain

$$
\begin{aligned}
c_{i} & =\sum_{m=1}^{s} \rho_{i}\left(r\left(a_{m}\right)^{-\frac{1}{2}} a_{m}\right) \rho_{i}\left(r\left(\bar{a}_{m}\right)^{-\frac{1}{2} a_{m}}\right) \\
& =\sum_{m=1}^{\delta} \rho_{i}\left(b_{m}\right) \overline{\rho_{i}\left(b_{m}\right)}>0,
\end{aligned}
$$

where $b_{m}=r\left(a_{m}\right)^{-\frac{1}{2}} a_{m}$ ranges over the standard elements in $H$.

The next two results, 8 and 9 , are fundamental in establishing the duality Theorem 12 . 


\section{Lemma}

Let $H=\left\{1=a_{1}, a_{2}, \ldots, a_{s}\right\}$ be an abelian hypergroup with coefficients in a splitting field $k$. The grouplikes $\left\{d=\rho_{1}, \rho_{2}, \ldots, \rho_{s}\right\}$ in $(k H) *$ have the following properties (where the structure constants are assumed nonzero in (iii) and (iv)):

(i) $d(1)=1$;

(ii) $\sum_{m=1}^{s} r\left(a_{m}\right)^{-1} \rho_{i}\left(a_{m}\right) \rho_{j}\left(a_{m}\right)=c_{j} \delta_{i j} ;$

(iii) $\sum_{m=1}^{s} h_{m} \rho_{m}\left(a_{i}\right) \rho_{m}\left(a_{j}\right)=\|H\|_{r}\left(a_{i}\right) \delta_{i j}$ where $h_{m}=c_{1} c_{m}^{-1}$;

(iv) $\sum_{m=1}^{s} h_{m} \rho_{m}\left(a_{i}\right) \rho_{m}\left(a_{j}\right) \rho_{m}\left(\bar{a}_{k}\right)=\|H\| r\left(a_{k}\right) n\left(a_{i}, a_{j}, a_{k}\right)$.

Proof. Statement $(i)$ is trivial while $(i i)$ is proved by analogy with $[6,4.1]$ using (7) and (8) above. Standard manipulations of matrices show that $(i i)$ and (iii) are equivalent.

To prove (iv), begin with its left-hand side. Then

$$
\begin{aligned}
\sum_{m} h_{m} \rho_{m}\left(a_{i}\right) \rho_{m}\left(a_{j}\right) \rho_{m}\left(a_{k}\right) & =\sum_{m} h_{m} \rho_{m}\left(a_{i}\right) \sum_{p} n\left(a_{j}, \bar{a}_{k}, \bar{a}_{p}\right) \rho_{m}\left(a_{p}\right) \\
& =\sum_{p} n\left(a_{j}, \bar{a}_{k}, \bar{a}_{p}\right) \sum_{m} h_{m} \rho_{m}\left(a_{i}\right) \rho_{m}\left(a_{p}\right) \\
& =n\left(a_{j}, \bar{a}_{k}, \bar{a}_{i}\right)\|H\| r\left(a_{i}\right) \text { by (iii) } \\
& =n\left(a_{j}, \bar{a}_{i}, \bar{a}_{k}\right)\|H\| r\left(a_{k}\right) \\
& =n\left(a_{i}, a_{j}, a_{k}\right)\|H\| r\left(a_{k}\right),
\end{aligned}
$$

the required identity.

\section{Proposition}

Let $H_{0}$ denote the hypergroup in $k H$ consisting of grouplikes, where $H$ is a finite abelian hypergroup with coefficients in a splitting field $k$. (Hence $\left.H_{0}=\left\{d(a)^{-1} a \mid a \in H\right\}.\right)$ If the structure constonts of $H$ are nonzero, then the coefficient of $\rho_{k}$ in $\rho_{i} \cdot \rho_{j}=M^{*}\left(\rho_{i} \otimes \rho_{j}\right)$ is 


$$
n_{0}^{*}\left(\rho_{i}, \rho_{j}, \rho_{k}\right)=c_{k}^{-1} \sum_{a \in H_{0}} r(a)^{-1} \rho_{i}(a) \rho_{j}(a) \rho_{k}(\bar{a})
$$

where $\left\{d=\rho_{1}, \rho_{2}, \ldots, \rho_{s}\right\}$ is the set of grouplikes in $(k H)^{*}$.

Proof. Let $b$ be a grouplike in $k H$. Then

$\sum_{k}\left\{c_{k}^{-1} \sum_{a \in H_{0}} r(a)^{-1} \rho_{i}(a) \rho_{j}(a) \rho_{k}(\bar{a})\right\} \rho_{k}(b)$

$$
\begin{aligned}
& =\sum_{a} r(a)^{-1} \rho_{i}(a) \rho_{j}(a) \sum_{k} c_{k}^{-1} \rho_{k}(\bar{a}) \rho_{k}(b) \\
& =r(b)^{-1} \rho_{i}(b) \rho_{j}(b) r(b) \text { by } 8(i i i) \\
& =M^{*}\left(\rho_{i} \otimes \rho_{j}\right)(b),
\end{aligned}
$$

the last step following from (1) since $b$ is grouplike. The proof is completed by using the fact that the grouplikes are bases for $k H$ and $(k H)^{*}$.

\section{Corollary}

$$
r^{*}\left(\rho_{k}\right)\left(=n_{0}^{*}\left(\rho_{k}, \bar{\rho}_{k}, \rho_{1}\right)\right)=h_{k}^{-1} \text {. }
$$

\section{1. $(k H) *$ as a double algebra}

Let $H_{0}$ denote the set of grouplikes in $k H$, where $H$ is a finite abelian hypergroup with coefficients in a splitting field $k$. Assume also that the structure constants for $H$ are nonzero. Define a map $a \mapsto a^{\#}$ from $H_{0}$ to $(k H) * *$ by

$$
a^{\#}(\rho)=\rho(a) \text { for } \rho \in(k H)^{*} \text {. }
$$

For $a \in H_{0}, a^{\#}$ is a grouplike and so the grouplikes span $(k H)^{* *}$. Turning to the structure constants $c_{i}^{*}$ of $(k H)^{*}$, they satisfy

$$
\begin{aligned}
c_{i}^{*} & =\sum_{m} r^{*}\left(\rho_{m}\right)^{-1} a_{i}^{\#}\left(\rho_{m}\right) a_{i}^{\#}\left(\bar{\rho}_{m}\right) \\
& =\sum_{m} h_{m} \rho_{m}\left(a_{i}\right) \rho_{m}\left(\bar{a}_{i}\right)=\|H\| r\left(a_{i}\right),
\end{aligned}
$$


the last step by 8 (iii), and hence are also nonvanishing.

Define $\theta_{1}^{*}:(k H)^{*} \rightarrow k$ as the $k$-linear map which sends each $\rho$ in $(k H)^{*}$ to the coefficient of $d$ when $\rho$ is written in terms of grouplikes. In view of Proposition 3, Theorem 4, and the definition of a splitting field, to show that $(k H)^{*}$ is a double algebra (that is, that it satisfies (Al) $-(A 7)$ of [5]), it only remains to check that

$$
\theta_{1}^{*}\left(\rho_{i} \bar{\rho}_{j}\right) \neq 0 \text { if and only if } \rho_{i}=\rho_{j} \text { for grouplikes } \rho_{i}, \rho_{j} \text {. }
$$

But 8 (ii) and Proposition 9 assure us that

$$
\begin{aligned}
\theta_{1}^{*}\left(\rho_{i} \bar{\rho}_{j}\right) & =n_{0}^{*}\left(\rho_{i}, \bar{\rho}_{j}, d\right)=c_{1}^{-1} \sum_{a \in H_{0}} r(a)^{-1} \rho_{i}(a) \rho_{j}(\bar{a}) \\
& =c_{1}^{-1} c_{i} \delta_{i j},
\end{aligned}
$$

and so $\theta_{1}^{*}$ has the desired property since the structure constants for $k H$ are nonzero.

Hence, under the above conditions, $(k H)^{*}$ is a double algebra with nonzero stmucture constants. Furthermore, $k$ is a splitting field for $(k H)^{*}$ in the sense that the grouplikes in $(k H)^{* *}$ are spanning. Thus $(k H)^{* *}$ is also a double algebra.

\section{Theorem}

Suppose that $H$ is a finite abelian hypergroup with coefficients in a splitting field $k$ and that the stmucture constants of $H$ are nonvanishing. Then the $k$-linear extension of the map $a \mapsto a^{\#}$ defined in the previous section is a double algebra isomorphism between $k H$ and $(k H) * *$,

Proof. Since the above map is easily seen to respect the degree and conjugation maps, we only need to check that $n_{0}^{* *}\left(a_{i}^{\#}, a_{j}^{f^{\prime \prime}}, a_{k}^{\#}\right)=n\left(a_{i}, a_{j}, a_{k}\right)$ for grouplikes $a_{i}, a_{j}, a_{k}$. Starting from the left hand side with Proposition 9, 


$$
\begin{aligned}
n_{0}^{* *}\left(a_{i}^{\#}, a_{j}^{\#}, a_{k}^{\#}\right)= & \left(c_{k}^{*}\right)^{-1} \sum_{m} r^{*}\left(\rho_{m}\right)^{-1} a_{i}^{\#}\left(\rho_{m}\right) a_{j}^{\#}\left(\rho_{m}\right) a_{k}^{\#}\left(\rho_{m}\right) \\
& \text { (where the surmation is over grouplikes in } \left.(k H)^{*}\right) \\
= & \|H\|^{-1} r\left(a_{k}\right)^{-1} \sum_{m} h_{m} \rho_{m}\left(a_{i}\right) \rho_{m}\left(a_{j}\right) \rho_{m}\left(a_{k}\right) \\
= & n\left(a_{i}, a_{j}, a_{k}\right) \text { by } 8(i v),
\end{aligned}
$$

as desired.

\section{Remarks}

The matrix $\left(\rho_{j}\left(a_{i}\right)\right)_{i, j}$, where $H=\left\{1=a_{I}, \ldots, a_{s}\right\}$ is a finite abelian hypergroup and the $\rho_{j}$ are the grouplikes in $(k H)^{*}$, is called the character table arising from $H$ and is stuaied in [6]. See also [1]. Note that if $G$ is a finite group then the character table of $G$ is the character table arising from $\hat{G}$. In this case formulae 8 (ii), (iii) reduce to well-known orthogonality relations on a (group) character table (see (31.13) and (31.11) of [2]), while 8 (iv) and 9 describe the product of two characters or two conjugacy classes respectively in terms of the entries of the character table (see (32.8) of [2] and (2.15) of [3]; these decompositions also appear as (10) and (12) below).

\section{The dual of a finite abelian hypergroup}

Let $H$ be a finite abelian hypergroup with coefficients in a splitting field $k$. Assume also that the structure constants of $k H$ are nonzero. The grouplikes in $k H$ are the elements $d(a)^{-1} a \quad(a \in H)$. Denote by $H_{0}^{*}=\left\{d=\rho_{1}, \rho_{2}, \ldots, \rho_{s}\right\}$ the hypergroup of grouplikes in $(k H)^{*}$. By results 8 and 9 above, the hypergroup structure of $H_{0}^{*}$ is described by the following formulat:

$$
\begin{aligned}
n_{0}^{*}\left(\rho_{i}, \rho_{j}, \rho_{k}\right) & =c_{k}^{-1} \sum_{a \in H} d(a)^{-1} r(a)^{-1} \rho_{i}(a) \rho_{j}(a) \rho_{k}(\bar{a}) ; \\
r_{0}^{*}\left(\rho_{i}\right) & =c_{k} c_{I}^{-1}=h_{i}^{-1} ; \\
d_{0}^{*}\left(\rho_{i}\right) & =1 .
\end{aligned}
$$


Now let $H_{1}^{*}=\left\{d=\xi_{1}, \xi_{2}, \ldots, \xi_{s}\right\}$ denote the hypergroup of standard elements of $(k H)^{*}$. As $\xi_{i}=h_{i}^{\frac{2}{2}} \rho_{i}=\left(c_{1} / c_{i}\right)^{p} \rho_{i}$, we have

$$
\begin{aligned}
n_{1}^{*}\left(\xi_{i}, \xi_{j}, \xi_{k}\right) & =h_{i}^{\frac{1}{2}} h_{j}^{\frac{1}{2}} h_{k}^{-\frac{1}{2}} c_{k}^{-1} \sum_{a \in H} d(a)^{-1} r(a)^{-1} \rho_{i}(a) \rho_{j}(a) \rho_{k}(\vec{a}), \\
r_{1}^{*}\left(\xi_{i}\right) & =1 \\
d_{1}^{*}\left(\xi_{i}\right) & =h_{i}^{\frac{1}{2}} .
\end{aligned}
$$

Finally, let $H_{2}^{*}=\left\{d=\eta_{1}, \eta_{2}, \ldots, n_{s}\right\}$ denote the hypergroup of classlike elements of $(k H)^{*}$ with $\eta_{k}=h_{k} \rho_{k}$. Then

$$
\begin{aligned}
n_{2}^{*}\left(\eta_{i}, \eta_{j}, \eta_{k}\right) & =h_{i} h_{j}\|t\|^{-1} \sum_{a \in H} d(a)^{-1} r(a)^{-1} \rho_{i}(a) \rho_{j}(a) \rho_{k}(\bar{a}), \\
r_{2}^{*}\left(\eta_{i}\right) & =h_{i}, \\
d_{2}^{*}\left(\eta_{i}\right) & =h_{i} .
\end{aligned}
$$

From the above formulae and the proposition of Section 7 we see that:

PROPOSITION. If $H$ has real coefficients in the sense of 1.0 of [5], then so do $H_{0}^{*}, H_{1}^{*}$, and $H_{2}^{*}$.

\section{Duality between $\hat{G}$ and $\bar{G}$}

Let $G$ be a finite group and let $\hat{G}$ be the (standard) hypergroup of irreducible complex characters of $G$, and $\bar{G}$ the (classlike) hypergroup of conjugacy classes. Then

$$
\bar{G} \cong \hat{G}_{2}^{*}, \quad \hat{G} \cong \bar{G}_{1}^{*} .
$$

\section{The main result}

Let $k$ be a field and $A_{k}$ the category whose objects are finite abelian hypergroups with coefficients in $k$ and which split over $k$, and whose morphisms are the hypergroup morphisms. If $H, L$ are objects in $A_{k}$ and $\phi: H \rightarrow L$ a morphism (equivalently, $\phi: k H \rightarrow k L$ a morphism), then its linear adjoint $\phi^{*}:(k L)^{*} \rightarrow(k H)^{*}$ is also a morphism. Let $H^{*}$ 
denote any hypergroup which generates $(k H)^{*}$.

We now state in full the duality theorem for finite abelian hypergroups. Routine manipulations take care of those steps in the proof not already established.

THEOREM. Suppose that $k$ is a subfiezd of $\mathbf{R}$. Then $A_{k}$ is equivalent to $A_{k}^{\mathrm{op}}$; more precisely, the pair $D$ of maps $\left(H \mapsto H^{*}\right.$, $\left.\phi \mapsto \phi^{*}\right)$ is a functor from $A_{k}$ to $A_{k}^{\text {op }}$ and the isomorphisms \# : $H \rightarrow H^{* *}$ constitute a natural equivalence from the identity functor for $A_{k}$ to $D^{2}$.

\section{Self-dual hypergroups}

There has been some interest in the literature in the question of isomorphism of the algebras $\bar{Z} \bar{G}$ and $\mathbb{Z} \hat{G}$ (see Thompson [8]). It seems natural to ask whether $\bar{G}$ and $\hat{G}$ are isomorphic as hypergroups (a stronger property) for any nonabelian finite group $G$.

(a) If $\bar{G}$ and $\hat{G}$ are isomorphic as hypergroups, then it is readily seen that there exists a bijection $\psi: \bar{G} \rightarrow \hat{G}$ such that $d(\psi(k))^{2}=\# K$, the cardinality of $K$, for conjugacy classes $K$ and that $\left.\psi\right|_{Z(G)}$ is a group isomorphism $Z(G) \rightarrow\left(G / G^{\prime}\right)^{\wedge}$. These conditions are rarely satisfied for nonabelian groups.

(b) A nonabelian group $G$ with the property $\bar{G} \cong \hat{G}$ is

$$
\begin{array}{r}
G=\left\langle\alpha_{1}, \alpha_{2}, \alpha_{3}\right|\left[\alpha_{i}, \alpha_{j}\right]=\beta_{6-(i+j)},\left[\alpha_{i}, \beta_{i}\right]=\left[\alpha_{i}, \beta_{j}\right]=\left[\beta_{i}, \beta_{j}\right] \\
\left.=\alpha_{i}^{2}=\beta_{i}^{2}=1(i, j=1,2,3, i \neq j)\right\rangle .
\end{array}
$$

It is a group of order 64 with $G / Z(G)$ and $G^{\prime}=Z(G)$ both elementary abelian of order 8 . (It appears as group number 144 of Hall and Senior [4] and is a stem group of the family $\left.\Gamma_{9}.\right)$ The fact that $\bar{G}$ and $\hat{G}$ are isomorphic hypergroups follows by inspection of the character table of $G$ and application of the following proposition.

PROPOSITION. Let $x_{1}, \ldots, x_{s}$ denote the complex irreducible 
characters of a finite group $G$ and $K_{1}, \ldots, K_{s}$ its conjugacy classes. Suppose that there exist permutations $\alpha, \beta \in \underline{S_{0}}$ such that:

$$
\begin{gathered}
d\left(x_{i}\right)^{-1} x_{i}\left(k_{j}\right)=d\left(x_{\alpha(j)}\right)^{-1} x_{\alpha(j)}\left(k_{\beta(i)}\right), \\
d\left(x_{\alpha(i)}\right)^{2}=h_{i}, \quad d\left(x_{j}\right)^{2}=h_{\beta(j)},
\end{gathered}
$$

for $i, j=1, \ldots, s$. Then $\bar{G}$ and $\hat{G}$ are isomorphic.

Proof. Since the grouplikes in $(\mathfrak{\leftarrow G})^{*}$ are of the form $x_{i} \mapsto x_{i}\left(K_{j}\right)$

for $i, j=1, \ldots, s, 8$ (iv) shows that

$$
n\left(x_{i}, x_{j}, x_{k}\right)=(\# G)^{-i} \sum_{m=1}^{s} h_{m} x_{i}\left(K_{m}\right) x_{j}\left(K_{m}\right) x_{k}\left(K_{m}\right) .
$$

Hence

$$
\begin{aligned}
n\left(x_{\alpha(i)}, x_{\alpha(j)},\right. & \left.x_{\alpha(k)}\right) \\
& =(\# G)^{-1} \sum_{m} h_{B(m)} x_{\alpha(i)}\left(K_{B(m)}\right) x_{\alpha(j)}\left(K_{B(m)}\right) x_{\alpha(k)}\left(\bar{K}_{B(m)}\right) .
\end{aligned}
$$

On the other hand, the grouplikes in $(\mathbb{C} \bar{G})^{*}$ are of the form $K_{i} \rightarrow d\left(x_{j}\right)^{-1} h_{i} x_{j}\left(K_{i}\right)$ for $i, j=1, \ldots, s$ and so this time 8 (iv) gives

$$
n\left(K_{i}, K_{j}, K_{k}\right)=h_{i} h_{j}(\# G)^{-1} \sum_{m} d\left(x_{m}\right)^{-1} x_{m}\left(K_{i}\right) x_{m}\left(K_{j}\right) x_{m}\left(K_{k}\right) .
$$

Under the given hypothesis, identities (11) and (12) show that the map $\phi: \mathbb{a} \bar{G} \rightarrow \mathbb{a} \hat{G}$, defined as the linear extension of $k_{i} \mapsto h_{i} d\left(x_{\alpha(i)}\right)^{-1} \chi_{\alpha(i)}$, is an isomorphism.

\section{References}

[1] Richard Brauer, "On pseudo groups", J. Math. Soc. Japan 20 (1968), 13-22.

[2] Charles W. Curtis, Irving Reiner, Representation theory of finite groups and associative algebras (Pure and Applied Mathematics, 11. Interscience [John Wiley \& Sons], New York, London, 1962). 
[3] Walter Feit, Characters of finite groups (Benjamin, New York, Amsterdam, 1967).

[4] Marshal.l Hall, Jr., James K. Senior, The groups of order $2^{n}(n \leq 6)$ (Macmillan, New York; Collier-Macmillan, London; 1964).

[5] J.R. McMullen, "An algebraic theory of hypergroups", Buzl. Austral. Math. Soc. 20 (1979), 35-55.

[6] J.R. McMullen and J.F. Price, "Reversible hypergroups", Rend. Sem. Mat. Fis. Mizano (to appear).

[7] Moss E. Sweedler, Hopf algebras (Benjamin, New York, 1969).

[8] John G. Thompson, "A non-duality theorem for finite groups", $J$. AZgebra 14 (1970), 1-4.

\footnotetext{
Department of Pure Mathematics,

University of Sydney,

Sydney,

New South Wales;

School of Mathematics,

University of New South Wales,

Kensington,

New South Wales.
} 